for racial and ethnic minority staff groups influenced by perceptions about institutional and structural discrimination. This included suspicions and fear around institutional pressure to be vaccinated, racial injustices in vaccine development and testing, religious or ethical concerns, and legitimacy and accessibility of vaccine messaging and communication.

Conclusion Drawing on a critical race perspective, we conclude that acknowledging historical and contemporary abuses of power is essential to avoid perpetuating and aggravating mistrust by de-contexualising hesitancy from the social processes affecting hesitancy, undermining efforts to increase vaccine uptake.

\section{OP36 IS RISK OF BIAS UNDERESTIMATED IN POORLY CONDUCTED SYSTEMATIC REVIEWS USING COCHRANE'S RISK OF BIAS TOOL FOR NON- RANDOMISED STUDIES? A META-EPIDEMIOLOGICAL REVIEW}

Erik Igelström*, Mhairi Campbell, Peter Craig, Srinivasa Vittal Katikireddi. MRC/CSO Social and Public Health Sciences Unit, University of Glasgow, Glasgow, UK

\subsection{6/jech-2021-SSMabstracts.36}

Background Systematic reviews of evidence frequently help inform research, clinical guidelines, and policy. Although randomised controlled trials (RCTs) are ideal, many areas of public health rely on evidence from non-randomised studies which are more susceptible to bias. 'Risk Of Bias In Non-Randomised Studies of Interventions' (ROBINS-I) is a widely used critical appraisal tool developed by Cochrane in 2016, which assesses risk of bias on an absolute scale, such that a low riskof-bias study is equivalent to a well-conducted RCT. ROBINSI has been seen as a major methodological innovation, but its complexity has led to concerns that it may be misapplied. We review for the first time how ROBINS-I is used in a sample of recent systematic reviews.

Methods Systematic reviews using ROBINS-I were identified by forward citation and keyword/abstract searches in six databases, restricted to January and February 2020. The review protocol was preregistered in PROSPERO (CRD42020170785). Reported ROBINS-I ratings and data on how ROBINS-I was used were extracted from each review. Methodological quality of reviews was assessed using AMSTAR 2 ('A MeaSurement Tool to Assess systematic Reviews'). Mixed-effects partial proportional odds regression was used to assess associations between review characteristics (e.g. methodological quality and industry funding) and risk-ofbias ratings. Screening and quality appraisals were conducted independently by two reviewers.

Results Of 181 hits, 124 reviews were analysed with data extracted on 1,344 included studies. Risk of bias was reported as serious/critical for $54.8 \%$ of included studies, most commonly due to confounding, but $8.0 \%$ reported low risk of bias. Poorly conducted reviews were more likely to report lower risk-of-bias ratings, with an apparent dose-response relationship. Compared to reviews with moderate/high AMSTAR 2 rating, odds of low risk-of-bias ratings were higher in lowquality reviews (odds ratio: 1.89 [95\% confidence interval: $0.36-9.94]$ ), and considerably higher in critically low-quality reviews (4.70 [1.01-21.78]). Competing interests and industry funding were not uniformly predictive of higher or lower ratings, although these analyses had low statistical power.
Deviations from the guidance of the tool were seen in $40.3 \%$ of studies, with $20.2 \%$ reporting ratings using a non-standard scale.

Discussion Systematic reviews conducted using Cochrane's recommended tool for non-randomised studies may misleadingly suggest a robust evidence base exists when used by reviewers without adequate epidemiological expertise. This may lead to misleading conclusions, especially for public health guidelines. Greater training and expertise are required to ensure that widespread use of the tool does not lead to an increase in misleading reviews.

\section{Friday 17 September}

\section{Session: Food \& Food Policy, $09.00-11.30$}

\section{OP37 CHILDHOOD CONSUMPTION OF ULTRA-PROCESSED FOODS AND LONG-TERM ADIPOSITY TRAJECTORIES: FINDINGS FROM A UK BIRTH COHORT STUDY}

${ }^{1}$ Kiara Chang*, 2,3,4 Neha Khandpur, ${ }^{2,3}$ Daniela Neri, ${ }^{5}$ Mathilde Touvier, ${ }^{6}$ Inge Huybrechts, ${ }^{1}$ Christopher Millett, ${ }^{1}$ Eszter Vamos. ${ }^{1}$ Public Health Policy Evaluation Unit, Imperial College London, London, UK; ${ }^{2}$ Department of Nutrition, University of Sao Paulo, Sao Paulo, Brazil; ${ }^{3}$ Center for Epidemiological Research in Nutrition and Health, University of Sao Paulo, Sao Paulo, Brazil, ${ }^{4}$ Department of Nutrition, Harvard T. H. Chan School of Public Health, Boston, US; ${ }^{5}$ INSERM U1153, Sorbonne Paris Nord University, Bobigny, France; ${ }^{6}$ Nutrition and Metabolism Branch, International Agency for Research on Cancer, Lyon, France

\subsection{6/jech-2021-SSMabstracts.37}

Background Worldwide consumption of ultra-processed foods is continued to rise. Growing evidence has linked ultra-processed food consumption with elevated risks of obesity, noncommunicable diseases, and mortality in adults. Associations between ultra-processed food consumption and long-term adiposity trajectories have never been investigated in children.

Methods Data were obtained from the Avon Longitudinal Study of Parents and Children (ALSPAC), a prospective birth cohort study conducted in Avon County, south-west England. Participating children with baseline dietary intakes collected using 3-day food diaries and repeated measures of adiposity outcome were included and followed up from ages 7 to 24 years (1998-2017). Adiposity outcomes included objectively assessed anthropometrics (body mass index, weight, waist circumference) and dual-energy X-ray absorptiometry measurements (fat and lean mass index, body fat percentage). All foods and drinks consumed were categorised according to the degree of processing applying the NOVA food classification system. Individual's consumption of ultra-processed foods was derived as a percentage of its weight contribution (gram per day) in the total diet and categorised into quintiles. Associations between quintiles of ultra-processed food consumption and trajectories of adiposity outcomes were evaluated using linear growth curve models and adjusted for study covariates.

Results A total of 9025 children $(49.6 \%$ female $)$ were followed up over a median (IQR) of 10.2 (5.2-16.4) years. Mean (SD) ultra-processed food consumption at baseline from the lowest to highest consumption quintiles was $23.2 \%$ (5.0\%), 34.7\% (2.5\%), 43.4\% (2.5\%), 52.7\% (2.8\%) and $67.8 \%(8.1 \%)$. Trajectories of body mass index, fat mass index, weight and waist circumference increased significantly 
by an additional $0.06(95 \% \mathrm{CI}, 0.04$ to 0.08$) \mathrm{kg} / \mathrm{m}^{2}, 0.03$ (95\% CI, 0.01 to 0.05$) \mathrm{kg} / \mathrm{m}^{2}, 0.20$ (95\% CI, 0.11 to 0.28 ) $\mathrm{kg}$ and 0.17 (95\% CI, 0.11 to 0.22$) \mathrm{cm}$ per year among those in the highest quintile of ultra-processed food consumption compared with their lowest quintile counterpart. Evidence of dose-response relationships were consistently observed with those in the two highest quintiles of ultra-processed food consumption showing significantly more rapid progression of body mass index, weight, and waist circumference.

Conclusion These findings provide important and novel evidence that higher consumption of ultra-processed foods is associated with more rapid progression of adiposity from childhood to early adulthood. Radical and effective public health actions that reduce children's exposure and consumption of ultra-processed foods and encourage greater consumption of minimally processed foods are urgently needed to counteract the growing burden of obesity in England and globally.

\section{OP38 FRUIT AND VEGETABLES FISCAL POLICIES FOR REDUCING CARDIOVASCULAR MORTALITY AND RELATED INEQUALITIES: A MODELLING STUDY IN A LARGE SOUTHERN EUROPEAN URBAN POPULATION}

${ }^{1}$ Carlos Fernández-Escobar*, ${ }^{2} J u l i a$ Díez, ${ }^{3}$ Alba Martínez-García, ${ }^{4}$ Beatriz González LópezValcárcel, ${ }^{5}$ Simon Capewell, ${ }^{2}$ Manuel Franco, ${ }^{5}$ Martin O'Flaherty. ${ }^{1}$ Escuela Nacional de Sanidad, Instituto de Salud Carlos III, Madrid, Spain; ${ }^{2}$ Departamento de Cirugía, Ciencias Médicas y Sociales, Universidad de Alcalá, Alcalá de Henares, Spain; ${ }^{3}$ Departamento de Enfermería Comunitaria, Medicina Preventiva y Salud Pública, Universidad de Alicante, Alicante, Spain; ${ }^{4}$ Departamento de Métodos Cuantitativos en Economía y Gestión, Universidad de Las Palmas de Gran Canaria, Las Palmas Gran Canaria, Spain; ${ }^{5}$ Department of Public Health and Policy, University of Liverpool, Liverpool, UK

\subsection{6/jech-2021-SSMabstracts.38}

Background Fruit and vegetable $(\mathrm{F} \& \mathrm{~V})$ intake is an important modifiable risk factor for cardiovascular disease (CVD). Fiscal policies have been recommended to increase F\&V consumption, but their potential impact on health and inequalities are not well described.

We therefore explored the potential benefits of five different fiscal policies on F\&V consumption, CVD mortality and associated socio-economic inequalities in Madrid, Spain (total population $\sim 6,700,000$ residents).

Methods A modelling study using a comparative risk assessment approach.

We compared five policy options:

Option 1) reducing F\&V Value Added Tax (VAT) from 4\% to $0 \%$;

Option 2) a $\mathrm{F} \& \mathrm{~V}$ price discount of $10 \%$;

Option 3) a targeted F\&V subsidy of 50\% for low-income families;

Option 4) a mass media campaign promoting F\&V consumption, and

Option 5) The combination of Options 3 and 4.

We computed F\&V intake, CVD deaths prevented or postponed (DPPs), and CVD mortality inequalities between quintiles of area-level socio-economic status (SES) in 2021-2035, compared to a no-intervention scenario based on observed trends in F\&V intake and CVD mortality. We used estimates for price elasticities, pass-through of policies, and F\&V effect measures from relevant sources. We conducted sensitivity analyses through 5,000 Monte Carlo iterations with wide margins using the statistical software R.
Results A combined mass media campaign and 50\% targeted discount could prevent or postpone approximately $700 \mathrm{CVD}$ deaths (95\% UI: $50-1000)$ in 2021-2035, representing a $1 \%$ reduction in total CVD mortality. A $50 \%$ targeted discount alone might generate some 450 DPPs, (95\%UI: $300-$ 700), a $10 \%$ universal subsidy approximately 300 DPPs, (95\% UI: 100 - 450), a mass media campaign some 200 DPPs, (95\%UI: 150 - 400), and 0\% VAT 100 DPPs, (95\%UI: $50-$ 200). Every option would modestly reduce socio-economic inequalities in CVD mortality, with the combined policy achieving the greatest reduction, approximately $10 \mathrm{DPPs}$ / 100,000 population between highest- and lowest-SES areas.

Conclusion Fiscal policies and mass media campaigns targeting F\&V consumption applied singly, or ideally in combination, could reduce CVD mortality and decrease social inequalities in a large southern European urban population.

\section{OP39 ESTIMATING GEOGRAPHICAL INEQUALITIES IN FRUIT AND VEGETABLE INTAKE IN LIVERPOOL, UK: A SPATIAL MICROSIMULATION APPROACH}

${ }^{1}$ Ellen Schwaller*, ${ }^{2}$ Mark Green, ${ }^{1}$ Christodolous Kypridemos, ${ }^{3}$ Grace Patterson, ${ }^{1}$ Martin O'Flaherty. 'Public Health, Policy and Systems, University of Liverpool, Liverpool, UK; ${ }^{2}$ Geography and Planning, University of Liverpool, Liverpool, UK; ${ }^{3}$ Epidemiology and Population Health, University of Liverpool, Liverpool, UK

\subsection{6/jech-2021-SSMabstracts.39}

Background Individual-level data of health outcomes and their drivers are rarely available at a neighbourhood-level. While national-level surveys may provide insights at a regional scale, these data do not help us understand how complex problems such as poor diet affect communities within cities where policies can be leveraged for better health outcomes. Spatial microsimulation (SMS) can be used to approximate individuallevel data for small areas. The aim of our study is to estimate small area fruit and vegetable intake for Liverpool (UK) to explore geographical inequalities in diet.

Methods Spatial microsimulation leverages individual level data and fits it to distinct spatial data. The fitting is based on selected constraint variables contained within both data sets. We perform the SMS using individual-level data extracted from the National Dietary and Nutritional Survey (2014-2018, $n=2018$ ) and aggregated data from 2011 UK Census at Lower Super Output Area (LSOA). Our outcome variable is daily servings of fruits and vegetables consumed. Constraint variables include age, sex, highest level of qualifications, self-reported health, and national statistics-socioeconomic classification. Constraints were chosen based on literature of key determinants of dietary predictors existing across both data sets. An iterative proportional fitting approach is executed in $\mathrm{R}$ to estimate fruit and vegetable intake for each LSOA. We use internal validation via model fit statistics and external validation through comparing estimates to an Eating Habits Survey $(n=1724)$ with partial representation of Liverpool.

Results We estimated that $25 \%$ of adults aged 16 and up in Liverpool meet their daily recommendation of fruit and vegetable intake (5 or more servings), with $7 \%$ consuming fewer than 1 serving a day. Fruit and vegetable consumption was unevenly distributed across the city, with consumption of 5 or more servings ranging from $18 \%$ to $37 \%$. There were higher levels of fruit and vegetable consumption among populations in the least deprived neighbourhoods with $35 \%$ meeting daily 